ACREDITAÇÃO DO ENSAIO DE IMPACTO CHARPY: ESTUDO DE CASO NO LAMEF

\title{
CHARPY IMPACT TESTING ACCREDITATION: LAMEF CASE STUDY
}

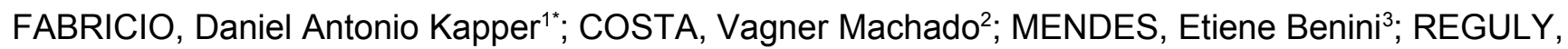 \\ Afonso; ; STROHAECKER, Telmo Roberto ${ }^{5}$
}

1,2,3,4,5 Universidade Federal do Rio Grande do Sul, Escola de Engenharia, Laboratório de Metalurgia Física.

Av. Osvaldo Aranha, 99/610, CEP 90035-190, Porto Alegre - RS, Brasil

(fone: +55 513308 4251; fax: +55 513308 3988)

\author{
* Autor correspondente \\ e-mail: danielkapper@gmail.com
}

Received 19 December 2014; received in revised form 5 January 2015; accepted 10 January 2015

\section{RESUMO}

O ensaio de impacto permite avaliar a energia absorvida por um corpo de prova padronizado de acordo com as normas ASTM E23 e ASTM E2298. O presente trabalho tem como objetivo relatar a adequação do Sistema de Gestão da Qualidade do Laboratório de Metalurgia Física (LAMEF) da UFRGS para a acreditação do ensaio de impacto em corpos de prova do tipo Charpy. Foi realizada uma análise dos requisitos gerenciais e técnicos da norma ABNT NBR ISO/IEC 17025 visando identificar quais eram os pontos que necessitavam adequação. Além disso, o ensaio foi submetido a uma auditoria externa que apontou algumas não conformidades. Após a realização das ações necessárias, o ensaio foi acreditado com sucesso, passando a fazer parte do escopo do LAMEF.

Palavras-chave: ABNT NBR ISO/IEC 17025; Sistema de Gestão da Qualidade; Laboratório de Ensaios; Ensaio de Impacto.

\begin{abstract}
The impact test evaluates the energy absorbed by a standardized test specimen in accordance with ASTM E2298 and ASTM E23 standards. This study aims to report the adequacy of the Quality Management System of Physical Metallurgy Laboratory (LAMEF) of UFRGS for the accreditation of the test impact on the Charpy type specimens. An analysis of ISO/IEC 17025 managerial and technical requirements has been performed to identify which points required adjustment. In addition, an external audit was executed, pointing some nonconformities. After performing the necessary actions, the impact test has been successfully accredited, being included in the LAMEF scope.
\end{abstract}

Keywords: ISO/IEC 17025; Quality Management System; Testing Laboratory; Impact Testing.

\section{INTRODUÇÃO}

O ensaio de impacto é um ensaio
dinâmico utilizado para análise do
comportamento de fratura de materiais. Esse
ensaio fornece a energia necessária para fraturar
uma amostra padronizada de acordo com as
normas ASTM E23 e BS EN ISO 148-1.
Entretanto, deve ser enfatizado que a energia de
impacto absorvida pelo corpo de prova fornece uma informação qualitativa de tenacidade à fratura, sendo que seus resultados podem ser somente comparados entre si ou com um valor determinado em uma especificação. Uma das principais funções do ensaio de impacto Charpy está em determinar se um material apresenta ou não uma transição dúctil-frágil com a variação da temperatura de ensaio e, caso isso ocorra, qual a faixa de temperatura que acontece este fenômeno (GARCIA et al., 2012). As Figuras 1 e 
2 apresentam exemplos de curvas de transição dúctil-frágil obtidas com a realização do ensaio Charpy.

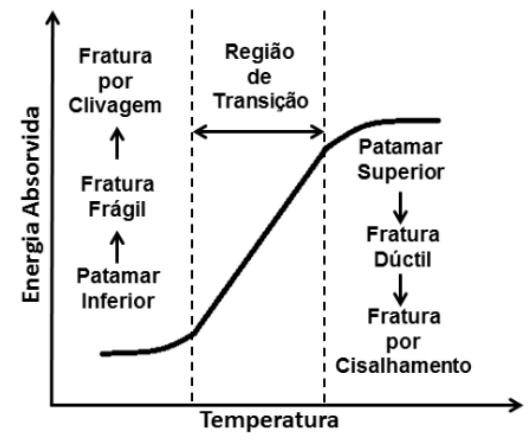

Figura 1. Curva típica de um material que apresenta uma transição dúctil-frágil significativa Fonte: CIMM (2014).

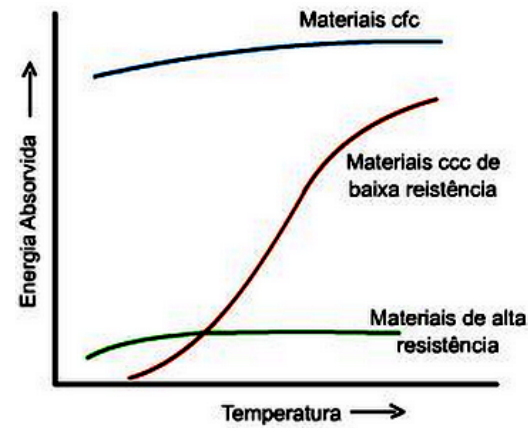

Figura 2. Comportamento de diferentes materiais submetidos ao ensaio de impacto Fonte: CIMM (2014).

A implantação de sistemas de gestão em ambiente laboratorial traz inúmeros benefícios, tais como: garantia da qualidade dos resultados e rastreabilidade das medições; aumento do número de serviços prestados e da satisfação dos clientes; melhoria da eficácia do laboratório, facilitando o trabalho e a integração de novos membros e a conscientização do pessoal; melhoria das atividades de pesquisa; aumento da qualificação da equipe de trabalho (ABDELFATAH, 2010; GROCHAU et al., 2010; RODIMA et al., 2005; ZAPATA-GARCIA et al., 2007).

A norma ABNT NBR ISO/IEC 17025 especifica requisitos gerais para a competência em realizar ensaios e/ou calibrações, cobrindo métodos normalizados, não normalizados e métodos desenvolvidos pelo próprio laboratório
(ABNT, 2005). A acreditação de ensaios e/ou calibrações de laboratórios que prestam esses serviços requer que o laboratório tenha um sistema de gestão implantado para todos os ensaios/calibrações de seu escopo, atendendo também a uma série de requisitos técnicos.

O Laboratório de Metalurgia Física (LAMEF) da Universidade Federal do Rio Grande do Sul (UFRGS) é acreditado desde 2010 na execução de ensaios mecânicos metalúrgicos. Em 2013, ocorreu um processo de aumento de escopo de acreditação, passando o LAMEF a ser acreditado, também, no ensaio de impacto Charpy. Nesse contexto, o objetivo do trabalho é descrever o processo de adequação do Sistema de Gestão da Qualidade do LAMEF para a acreditação do ensaio de impacto em corpos de prova do tipo Charpy, assim como as não conformidades encontradas e as ações corretivas tomadas até a consolidação do aumento de escopo.

\section{MATERIAL E MÉTODOS}

O laboratório possui um equipamento para ensaio de impacto Charpy da marca Instron modelo SI-1D3 com capacidade de $400 \mathrm{~J}$ e velocidade de impacto do pêndulo igual a 5,19 $\mathrm{m} / \mathrm{s}$ (Figura 3). A leitura da energia absorvida é através de escala analógica.

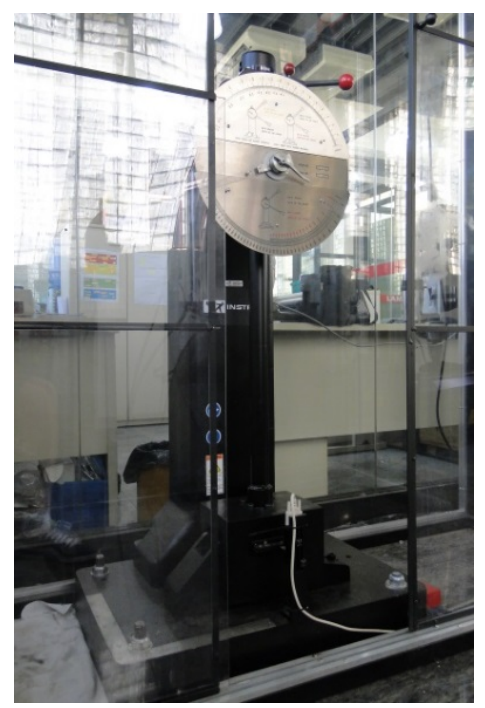

Figura 3. Equipamento utilizado para ensaio de impacto Charpy

Os corpos de prova utilizados para o ensaio de impacto Charpy devem ser retirados do material conforme a especificação de 
aplicação, tendo suas dimensões padronizadas pelas normas ASTM E23 e BS EN ISO 148-1. A Figura 4 apresenta as dimensões padronizadas pelas normas e os 3 tipos de entalhes possíveis, sendo o entalhe do tipo A o mais utilizado.

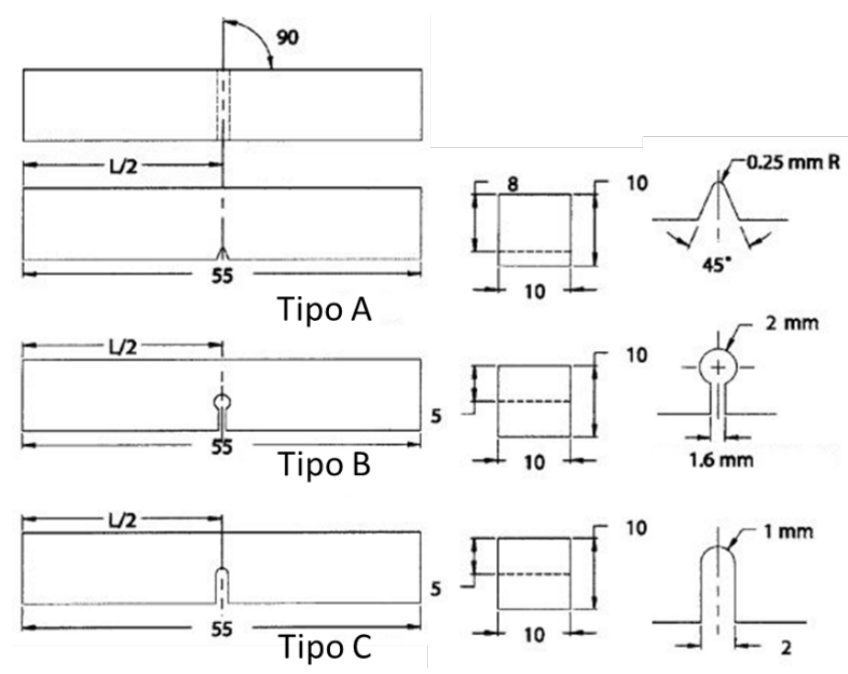

Figura 4. Tipos de corpos de prova para ensaio de impacto Charpy

Fonte: ASTM (2007).

As etapas seguidas no processo para inclusão do ensaio de impacto no escopo de acreditação são apresentadas na Figura 5. A Adequação Gerencial e a Adequação Técnica consistiram em adaptar-se aos requisitos do item 4 e 5 da ABNT NBR ISO/IEC 17025, focando no ensaio de impacto Charpy. Após essa adequação inicial, o LAMEF passou por Auditoria Externa pela Coordenação Geral de Acreditação (Cgcre) do Instituto Nacional de Metrologia, Qualidade e Tecnologia (Inmetro) para o aumento de escopo e identificação de não conformidades, em agosto de 2013. Para cada não conformidade encontrada, foi realizada investigação de causa, utilizando-se o método dos 5 porquês (CYGER et al., 2014), para tomada de Ações Corretivas. Após a tomada de tais ações, foram enviadas evidências das mesmas para a Cgcre em um prazo de 90 dias após a auditoria.

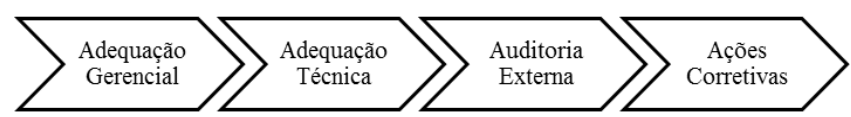

Figura 5. Etapas de trabalho

\section{RESULTADOS E DISCUSSÃO:}

A aplicação de cada uma das etapas descritas na Figura 5 é apresentada nas subseções a seguir.

\section{Adequação Gerencial}

Com um Sistema de Gestão da Qualidade implementado desde 2008 e acreditado pela Cgcre/Inmetro desde 2010, as adequações necessárias em termos gerenciais foram pequenas. O Manual da Qualidade tem como escopo "Ensaios Mecânicos", não sendo necessária assim nenhuma alteração, já que o Ensaio de Charpy está inserido neste contexto.

O procedimento técnico e os formulários específicos criados foram inseridos na Lista Mestra e disponibilizados para uso no software de controle de documentos utilizados pelo laboratório.

O ensaio foi acompanhado e avaliado durante o processo de Auditoria Interna. O Relatório gerado foi encaminhado à Cgcre/Inmetro para análise junto com os demais documentos necessários para a extensão de escopo. Portanto, em termos de adequações gerenciais foi necessário fazer poucas mudanças no Sistema.

\section{Adequação Técnica}

Para operação do equipamento, foi elaborado um procedimento interno baseado na norma ASTM E23. O procedimento descreve passo a passo como realizar o ensaio de impacto e os respectivos cuidados para realização dos ensaios.

Ao receber as amostras de Charpy, as mesmas são identificadas pelo número de solicitação interna e dígito com identificação unívoca nas duas extremidades da amostra. Após a ruptura, as partes rompidas são unidas e armazenadas por pelo menos 30 dias.

Foram registradas através de declaração do coordenador técnico do Laboratório as pessoas capacitadas e autorizadas para a execução do ensaio de impacto.

Antes da realização dos ensaios, os corpos de prova têm seus entalhes medidos em um projetor de perfil e registrados em um formulário de ensaio. Neste formulário também

PERIÓDICO TCHÊ QUÍMICA • www.periodico.tchequimica.com • Vol. 12 N. 23 - ISSN 1806-0374 (impresso) • ISSN 1806-9827 (CD-ROM) • ISSN 2179-0302 (meio eletrônico) 
são registradas as dimensões de espessura e largura das amostras, com o objetivo de certificar que as dimensões estão de acordo com o dimensional informado pela norma, além de registrar outras informações adicionais, tais como: orientação de retirada dos corpos de prova, temperatura de ensaio, tipo de material e energia absorvida no ensaio para cada amostra.

O equipamento onde o ensaio é executado (Figura 3) recebeu uma identificação e seus dados foram registrados em um Sistema de Gerenciamento de Equipamentos. Para garantir a rastreabilidade das medições realizadas, a máquina de ensaios é calibrada periodicamente.

Para atender ao requisito relacionado à incerteza de medição, foi elaborada uma planilha eletrônica (Figura 6) segundo os passos do Guia para Expressão da Incerteza de Medição (JCGM, 2008). Como a leitura da energia absorvida é direta e analógica, foram consideradas como fontes de incerteza a resolução do equipamento, o desvio padrão de repetitividade entre as amostras e a incerteza herdada do certificado de calibração.

\section{Auditoria Externa}

Em agosto de 2013, o LAMEF passou por auditoria pela Cgcre/Inmetro. Foram relatadas as não conformidades descritas na Tabela 1.

\section{Ações Corretivas}

Foi realizada investigação da causa raiz das não conformidades, para a tomada de ações corretivas. No caso da não conformidade número 01, a ação tomada foi elaborar um documento com uma tabela comparativa entre a incerteza de medição dos equipamentos (incluindo a máquina de impacto) e as normas de ensaio correspondentes. No caso da norma ASTM E23, havia exigência de erro máximo de $0,75 \%$ no total de perda por atrito, sendo que o equipamento possui erro de $0,422 \%$, estando este valor dentro do critério.

Para a não conformidade 02 , não havendo disponibilidade de ensaio de proficiência de impacto após a auditoria, o LAMEF organizou, em parceria com a Rede Metrológica RS, um programa de comparação interlaboratorial para o referido ensaio. Os resultados obtidos pelo LAMEF para o parâmetro energia absorvida foram considerados satisfatórios.

No caso da não conformidade 03 , foi realizada uma comparação intralaboratorial para o ensaio de impacto variando-se o operador. Para análise do resultado, utilizou-se o método do erro normalizado, obtendo-se um resultado satisfatório. Além disso, identificou-se que o procedimento de garantia da qualidade do laboratório não estava claro quanto à necessidade de controle de qualidade para todos ensaios, nem sobre a forma de execução. Assim, o procedimento foi revisado, e realizou-se um treinamento sobre a nova metodologia de monitoramento da qualidade.

Para a não conformidade número 04 , o procedimento de ensaio foi revisado, prevendo o método de execução para ensaios a baixa temperatura. Foi realizado, também, treinamento em tal procedimento.

Por fim, para a última não conformidade, foi projetada uma cuba criogênica (Figura 7) para permitir o resfriamento das amostras e a execução de ensaios a baixa temperatura. A aquisição da temperatura é realizada por um medidor de temperatura, o qual passou a ser calibrado para a faixa de $-40^{\circ} \mathrm{C}$ até $120^{\circ} \mathrm{C}$.
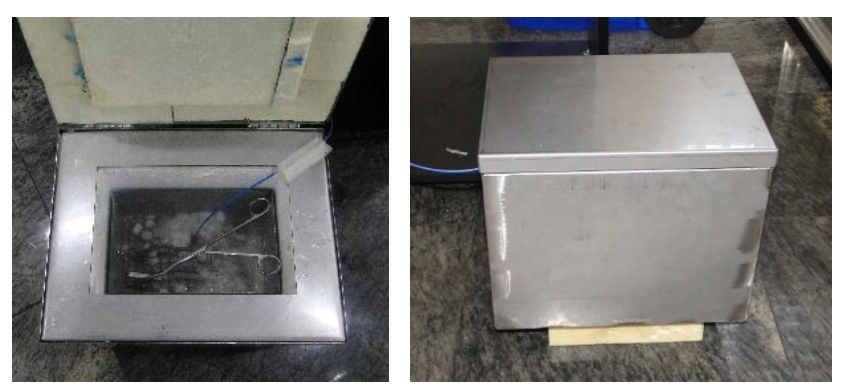

Figura 7. Cuba criogênica

\section{CONCLUSÕES:}

O trabalho descreveu o processo de aumento de escopo de acreditação do Laboratório de Metalurgia Física visando abranger o ensaio de impacto em corpos de prova tipo Charpy. Após a realização de adequações gerenciais e técnicas, o LAMEF passou por auditoria externa, na qual foram identificadas não conformidades.

Após a tomada de ações corretivas para eliminar a causa raiz dessas não conformidades, as evidências foram submetidas ao Inmetro. Ao 
final, consolidou-se o aumento de escopo do LAMEF, incluindo o ensaio de impacto Charpy, na faixa de temperatura de $-40^{\circ} \mathrm{C}$ até a temperatura ambiente.

\section{AGRADECIMENTOS:}

Os autores agradecem ao Eng. Bill Paiva dos Santos e ao Eng. Jefferson Haag pelo auxílio nas atividades relacionadas à acreditação do ensaio de impacto, e à Alta Direção do LAMEF pelo apoio nesse processo.

\section{REFERÊNCIAS:}

55. Abdel-Fatah, H. T. M. ISO/IEC 17025 accreditation: between the desired gains and the reality. The Quality Assurance Journal. 2010, 13, 21-27.

56. ABNT. NBR ISO/IEC 17025: Requisitos gerais para a competência de laboratórios de ensaio e calibração. Associação Brasileira de Normas Técnicas: Rio de Janeiro, 2005.

57. ASTM. ASTM E23: Standard Test Methods for Notched Bar Impact Testing of Metallic Materials. American Society for Testing and Materials: Pennsylvania, 2007.

58. CIMM. Temperatura de transição. Disponível em: www.cimm.com.br/portal/material_didatico/65 80-temperatura-de-transicao. Acessada em: 02 setembro 2014.

59. Cyger, M.; Ducceschi, E.; Barry, K.; Siteanu, L. Determine the Root Cause: 5 Whys. Disponível em: www.isixsigma.com/toolstemplates/cause-effect/determine-root-cause5-whys. Acessada em: 04 setembro 2014.

60. Garcia, A.; Spim, J.; Santos, C. A. Ensaio dos Materiais. 2 ed., LTC: Rio de Janeiro, 2012.

61. Grochau, I. H.; Ferreira, T. A.; Ferreira, J. P.; Caten, C. S. T. Implementation of a quality management system in university test laboratories: a brief review and new proposals. Accredit. Qual. Assur. 2010, 15, 681-689.

62. JCGM. Evaluation of measurement data Guide to the expression of uncertainty in measurement (GUM), 1st ed. Joint Committee for Guides in Metrology: 2008.

63. Rodima, A. et al. ISO 17025 quality system in a university environment. Accredit. Qual. Assur. 2005, 10, 369-372.

64. Zapata-Garcia, D.; Llauradó, M.; Rauret, G. Experience of implementing ISO 17025 for the accreditation of a university testing laboratory. Accredit. Qual. Assur. 2007, 12, 317-322.

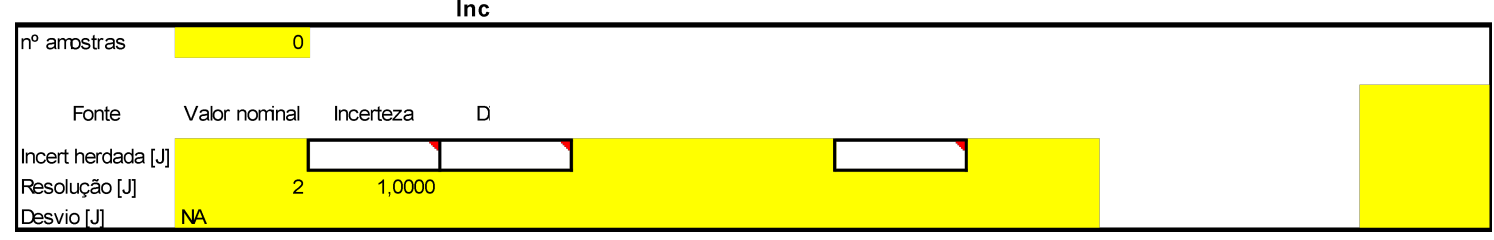

Figura 6. Planilha de incerteza de medição do ensaio de impacto 
Tabela 1. Não conformidades da auditoria externa relacionadas ao ensaio de impacto Charpy

\begin{tabular}{|c|c|c|}
\hline $\mathbf{N}^{\mathbf{0}}$ & Descrição da não conformidade & Requisito \\
\hline 01 & $\begin{array}{c}\text { O LAMEF não garante que os equipamentos atendem às } \\
\text { especificações da norma pertinente. Evidência: a análise crítica de } \\
\text { calibração não inclui a comparação da incerteza relatada nos } \\
\text { certificados de calibração com a incerteza especificada nas normas } \\
\text { de ensaio }\end{array}$ & $\begin{array}{l}\text { Item } 5.5 .2 \text { da } \\
\text { ABNT NBR } \\
\text { ISO/IEC } 17025\end{array}$ \\
\hline 02 & $\begin{array}{c}\text { O Laboratório não apresentou evidência de participação } \\
\text { satisfatória em ensaio de proficiência nos últimos dois anos em } \\
\text { partes significativas do seu escopo do pedido de extensão, apesar } \\
\text { da disponibilidade dos mesmos. }\end{array}$ & $\begin{array}{l}\text { Item } 9.1 .5 .1 \text { da } \\
\text { NIT-DICLA-026, } \\
\text { rev. } 08\end{array}$ \\
\hline 03 & $\begin{array}{l}\text { O laboratório não evidenciou dados de controle de qualidade para } \\
\text { monitorar os ensaios do escopo, incluindo o ensaio de impacto } \\
\text { Charpy. }\end{array}$ & $\begin{array}{l}\text { Item } 5.9 .1 \mathrm{da} \\
\text { ABNT NBR } \\
\text { ISO/IEC } 17025\end{array}$ \\
\hline 04 & $\begin{array}{l}\text { O procedimento de ensaio GEM PE } 013 \text { rev } 02 \text { não faz menção ao } \\
\text { ensaio de impacto Charpy em baixas temperaturas. }\end{array}$ & $\begin{array}{l}\text { Item } 5.4 .2 \text { da } \\
\text { ABNT NBR } \\
\text { ISO/IEC } 17025\end{array}$ \\
\hline 05 & $\begin{array}{l}\text { O laboratório não possui equipamentos auxiliares (dispositivos de } \\
\text { resfriamento de corpos de provas) para realizar ensaios de impacto } \\
\text { Charpy em baixas temperaturas }\end{array}$ & $\begin{array}{l}\text { Item } 5.5 .1 \mathrm{da} \\
\text { ABNT NBR } \\
\text { ISO/IEC } 17025\end{array}$ \\
\hline
\end{tabular}

PERIÓDICO TCHÊ QUÍMICA • www.periodico.tchequimica.com • Vol. 12 N. 23. - ISSN 1806-0374 (impresso) • ISSN 1806-9827 (CD-ROM) • ISSN 2179-0302 (meio eletrônico)

(C) 2015. Porto Alegre, RS. Brasil

The Periódico Tchê Química (ISSN: 1806-0374; 2179-0302) is an open-access journal since 2004. Journal DOI: 10.52571/PTQ. http://www.tchequimica.com. This text was introduced in this file in 2021 for compliance reasons.

OPEN ACCESS. This article is licensed under a Creative Commons Attribution 4.0 (CC BY 4.0) International License, which permits use, sharing, adaptation, distribution, and reproduction in any medium or format, as long as you give appropriate credit to the original author(s) and the source, provide a link to the Creative Commons license, and indicate if changes were made. The images or other third-party material in this article are included in the or exceeds the permitted use, you will need to obtain persis 